\title{
Adaptive Predictive Robust Control for Fuel Cells Hybrid Vehicles
}

\author{
Lucas Nieto Degliuomini ${ }^{a}$, David Zumoffen ${ }^{a, b}$, Marta Basualdo $^{a, b}$, Diego Feroldi $^{a}$ and Jordi Riera ${ }^{c}$ \\ Computer Aided for Process Eng. Group (CAPE-GIAIP) - CIFASIS-CONICET-UNR \\ 27 de Febrero 210 bis, S2000EZP Rosario, Argentine. \\ Phone: +54(0)341 423-7248 ext 301, Fax: +54(0)341 482-1772 \\ ${ }^{b}$ Universidad Tecnológica Nacional (UTN) - FRRo. Rosario, Argentine. \\ ${ }^{c}$ Institut de Robòtica i Informàtica Industrial (CSIC-UPC), Barcelona, Spain \\ basualdo,zumoffen,nieto,feroldi@ cifasis-conicet.gov.ar, riera@iri.upc.edu
}

\begin{abstract}
The transient behavior of a Polymer Electrolyte Membrane Fuel Cell System (PEMFCS) under an efficient Adaptive Predictive Control with Robust Filter (APCWRF) is analyzed. This control scheme is tested to evaluate its performance when sudden changes in the load occur. It is produced by the demands of the electric motor of a hybrid vehicle, powered by a PEMFC and a supercapacitor bank to fulfil Standard Driving Cycles. The objective of the proposed advanced strategy is to control the oxygen excess ratio in the cathode to improve the system efficiency and to ensure a safe operation for the PEM. Several results through a simulation environment are presented. They are useful for showing the potentiality of the APCWRF for the proposed exigent scenarios .
\end{abstract}

Keywords: Predictive Adaptive Robust Control, Fuel Cell Hybrid Vehicles, Energy Management Strategies, Energy Storage

\section{INTRODUCTION}

Fuel Cell Hybrid Vehicles (FCHV) are considered the most attractive long-term option for passenger cars. Hybridization in FCHV consists in adding a supplementary energy storage system (e.g., a battery and/or a supercapacitor bank) to the primary power source (i.e., the fuel cell) in order to adequate optimally the energy generation to the consumption. This procedure has important advantages, allowing a greater reduction of the hydrogen consumption.

Some works have appeared for Polymer Electrolyte Membrane Fuel Cell Systems (PEMFCS) connected to diverse electric motors devices $[1,2,3,4,5]$. Recently, in [6] has been presented a preliminary plantwide control structure for the process of hydrogen production from bio-ethanol to be used in a PEMFCS, accounting only steady-state information.
The focus of this paper is on controlling indirectly the PEMFCS net power output, which is considered as a performance variable. Therefore, the control problem discussed here consists on the regulation of the air (i.e. oxygen) supply to the fuel cell cathode, which is the controlled variable, even though a wide range of load disturbances. The optimal value of the compressor motor voltage, $V_{C M}$, (manipulated variable) which maximizes the PEMFCS net power, $P_{n e t}$ at each level of stack current is adopted from [7].

In this paper, it is specifically designed an APCWRF combined with a feedforward action for improving the regulation behavior when the disturbances are produced according to four standard and well known driving cycles requirements. Finally, several results are presented through a simulation setup, which are developed to show the behavior of a fuel cell hybrid vehicle working under these speed profiles, representing urban and highway scenarios. These tests are widely used in the literature because they are helpful to rigourously evaluate the performance and emissions of vehicles [8].

\section{THE MODEL}

The models of fuel cell systems describe quantitatively the physical and electrochemical phenomena that take place into the cells. In spite of having many models that study the cells in stationary state there are few dynamic models. In the work of Pukrushpan et al. [9] a dynamic model for PEMFCS that is suitable for the control study has been developed. The model captures the transitory behavior of the air compressor, the gasses filling dynamics (in the cathode and in the anode), and the effect of the membrane humidity. These variables affect the cell voltage and, therefore, 
the efficiency and the output power. The polarization curve in this model is a function of the hydrogen and oxygen partial pressures, the stack temperature and the membrane water content. This allows to evaluate the effect of variations of oxygen concentration and membrane humidity in the output voltage, which are necessary to make the control during transitory operation.

The model in [9] contains four main subsystems that interact with each other: $i$ ) the FC voltage subsystem, ii) the membrane hydration subsystem, iii) the cathode flow subsystem, and $i v$ ) the anode flow subsystem. The spatial variation of parameters is not considered and, thus, they are treated as lumped parameters. On the other hand, the time constants of the electrochemical reactions are in the order of magnitude of $10^{-19} \mathrm{~s}$. Thus, for control purpose, these time constants can be assumed negligible compared to other constants much slower: temperature $\left(10^{2} \mathrm{~s}\right)$ and dynamics of volume filling $\left(10^{-1} \mathrm{~s}\right)$.

The model takes into account the following nine states: 1 . the mass of oxygen in the cathode, $m_{\mathrm{O}_{2}}$ $(\mathrm{kg}) ; 2$. the mass of nitrogen in the cathode, $m_{N_{2}}$ $(\mathrm{kg}) ; 3$. the mass of water in the cathode, $m_{w, C a}(\mathrm{~kg})$; 4. the mass of hydrogen in the anode, $m_{H_{2}}(\mathrm{~kg}) ; 5$. the mass of water in the anode, $m_{w, A n}(\mathrm{~kg}) ; 6$. the compressor speed, $\omega_{c p}(\mathrm{rad} / \mathrm{s}) ; 7$. the supply manifold pressure, $p_{S M}(\mathrm{~Pa})$; 8. the mass of air in the supply manifold, $m_{S M}(\mathrm{~kg})$; 9 . the return manifold pressure, $p_{R M}(\mathrm{~Pa})$.

\section{CONTROL PROBLEM FORMULATION}

The control problem discussed in the present work focuses on the regulation of the air (i.e. oxygen) supply to the cathode. The oxygen excess ratio $\lambda_{O_{2}}$ can be defined as the ratio between the flow oxygen entering the cathode and the flow oxygen that reacts, $\lambda_{\mathrm{O}_{2}}=W_{\mathrm{O}_{2}, \text { in }} / W_{\mathrm{O}_{2}, \text { react }}$. In [7], it is concluded that there is an optimal oxygen excess ratio that produces a maximum net power for each current. Consequently, the compressor motor voltage, $\nu_{C M}^{o p t}$, which maximizes the stack net power, $P_{n e t}^{\max }$ can be approximated by the following expression:

$$
\nu_{C M}^{o p t}=-1.252 \cdot 10^{-3} I_{s t}^{2}+5.31 \cdot 10^{-2} I_{s t}+42.83
$$

However, usual control problems associated with feed-forward action such as offset existence occurred. Other important issue to be considered is that when the current demand from the vehicle suddenly increases, the oxygen consumption in the cathode increases too producing that the oxygen partial pressure drops. The accompanying drop in the $\mathrm{FC}$ voltage may lead to a short circuit and/or membrane damage. The phenomenon is known as oxygen starvation. To prevent this from happening, the air supply must be promptly increased to replenish the cathode with oxygen. Also, the hydrogen supplied to the anode must be quickly adjusted to ensure a minimal pressure difference across the FC membrane. A similar control of the $\mathrm{FC}$ system is required during a sudden drop in the stack current.

\section{A. Adaptive Predictive Control with Robust Filter (APCWRF)}

The control structure used here involves a commutation between a linear time-varying robustness filter (RF) in the feedback path of the control loop and an adaptive predictive controller (APC) (Fig. 1). The decision of which of both modes has to work is based on specific indicators deeply described in [10]. They are closely related to the operation conditions, which are checked every sampling time.

The strategy is developed in a modular way composed by two stages. First, the APC provides good asymptotic performance. Second, the adaptive predictive robustness filter APRF is applied if APC is unable to provide a good performance when sudden changes take place.

The predictive controller structure, when there is no constraints, can be obtained by minimizing the energy criterion in (2) applied at every step k.

$$
J(k)=\sum_{i=N_{1}}^{N_{2}} \alpha_{i}^{2} e^{2}(k+i)+\sum_{i=0}^{N_{u}-1} \beta_{i}^{2} \bar{u}^{2}(k+i)
$$

where $e(k)$ is a tracking error between a desired trajectory $y_{r}(k)$ and the predicted system output $\hat{y}(k)$ evaluated on a so-called prediction horizon $\left[N_{1}, N_{2}\right]$ via model, $y(k)$ and $u(k)$ are the past values of the system output and control action, respectively, and $\bar{u}(k)$ is the future control action calculated over the so-called control horizon $\left[0, N_{u}-1\right]$.

Considering the FIR model and the process operation point $\left[u_{00}, y_{00}\right]$ it can be expressed the model prediction of the plant as follows:

$$
\hat{y}(k+i)=\sum_{j=1+d}^{N} \hat{g}(j) u(k+i-j)+\hat{c g} l+\hat{\eta}(k+i)
$$

where $u(k+i)$ is the control signal, $\hat{y}(k+i)$ the model prediction, $\hat{\eta}(k+i)=y(k)-\hat{y}(k)$ the plantmodel mismatch (disturbances estimation) and $\hat{c g l}=$ $y_{00}-\sum_{j=d+1}^{N} \hat{g}(j) u_{00}$ the plant parameter, for $i=$ $N_{1} \ldots N_{2}$. 


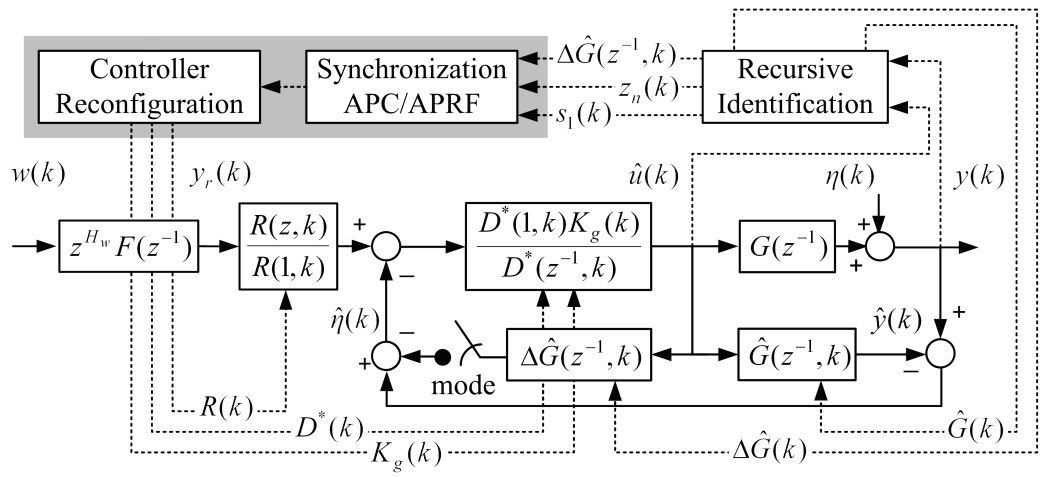

Fig. 1. Structure of the Adaptive Predictive Control with Robustness Filter (APCWRF) structure

An online adaptation of the linear FIR (3) can be done by using any recursive least square algorithm. Note that this adaptation also updates (2). This control policy is called APC. On the other hand, if the FIR model is parameterized as

$\hat{y}(k)=\sum_{i=1}^{N} \hat{\triangle g}(i) u(k-i)+\sum_{i=1}^{N} g_{0}(i) u(k-i)+c g l+\eta(k)$

In this case, the plant-model mismatch $\hat{\triangle g}(i)$ is adapted rather than the completed FIR model. This model structured generates the control policy known as APRF.

Finally, both control methodologies has been suitably synchronized as it is shown in Fig. 1 and called APCWRF. In the APC case (mode=0), the complete FIR model $\left(\hat{G}\left(z^{-1}\right)\right)$ and the controller parameters $\left(R(z), D^{*}\left(z^{-1}\right), K_{g}\right)$ are updated. On the other hand, in the APRF case (mode=1) both the plantmodel mismatch $\hat{\triangle g} g(i)$ and the static gain $K_{g}$ are adapted. The controller parameters in this case are the nominal values: $R_{0}(z), D_{0}^{*}\left(z^{-1}\right)$ given by the nominal FIR model $G_{0}\left(z^{-1}\right)$. For more details on this methodology see $[5,11,12]$.

\section{B. Energy Management Strategy (EMS)}

In the FCHV in study, the powertrain is composed by the PEMFCS and an Energy Storage System (ESS) composed by a supercapacitor bank in order to increase the efficiency of the integrated system (Fig. 2). Therefore, the hydrogen economy can be improved through hybridization. In this work, the power split between the PEMFCS and the ESS to power the FCHV is done through an Energy Management Strategy (EMS) based on the knowledge of the efficiency map of fuel cell and the state of charge on the supercapacitor bank.
The EMS operates the FCS preferably in its point of maximum efficiency in order to improve the hydrogen economy [13]. The operating point of the FCS is determined based on the the actual power demand and the state of energy of the ESS. The FCS power command is determined according to the following rules. If the load power is

$$
P_{f c s, l o} \cdot \eta_{B} \leq P_{\text {load }}(k) \leq P_{f c s, h i} \cdot \eta_{B}
$$

and, the $S o E$ is

$$
\operatorname{SoE}_{l o} \leq \operatorname{SoE}(k) \leq \operatorname{SoE}_{h i},
$$

where $P_{f c s, h i}$ is

$$
P_{f c s, h i}=P_{f c s, \max } \cdot \eta_{B} \cdot X_{f c s, h i},
$$

and $X_{f c s, h i}$ is a fraction of the maximum FCS power; then, the FCS is operated in its point of maximum efficiency:

$$
P_{f c s}(k)=P_{f c s, \text { max }} \text { eff } .
$$

The remaining power to achieve the load demand flows from the ESS if $P_{\text {load }}(k)>P_{f c s, \text { max eff }}$ (discharging mode):

$$
\begin{aligned}
P_{\text {ess }}(k)= & \min \left\{\frac{\left(P_{\text {load }}(k)-P_{f c s}(k) \cdot \eta_{B}\right)}{\eta_{B / B} \cdot \eta_{\text {ess }}},\right. \\
& \left.\left(\operatorname{SoE}(k)-S o E_{\text {min }}\right) \cdot k_{\text {ess }}\right\}
\end{aligned}
$$

or flow to the ESS if $P_{\text {load }}(k)<P_{f c s, \text { max eff }}$ (charging mode):

$$
\begin{aligned}
& P_{\text {ess }}(k)= \\
& =-\min \left\{\left|P_{\text {load }}(k)-P_{f c s}(k) \cdot \eta_{B}\right| \cdot \eta_{\text {ess }} \cdot \eta_{B / B},\right. \\
& \left.\quad\left|\operatorname{SoE}(k)-S o E_{\text {max }}\right| \cdot k_{\text {ess }}\right\} \quad \text { (10) }
\end{aligned}
$$




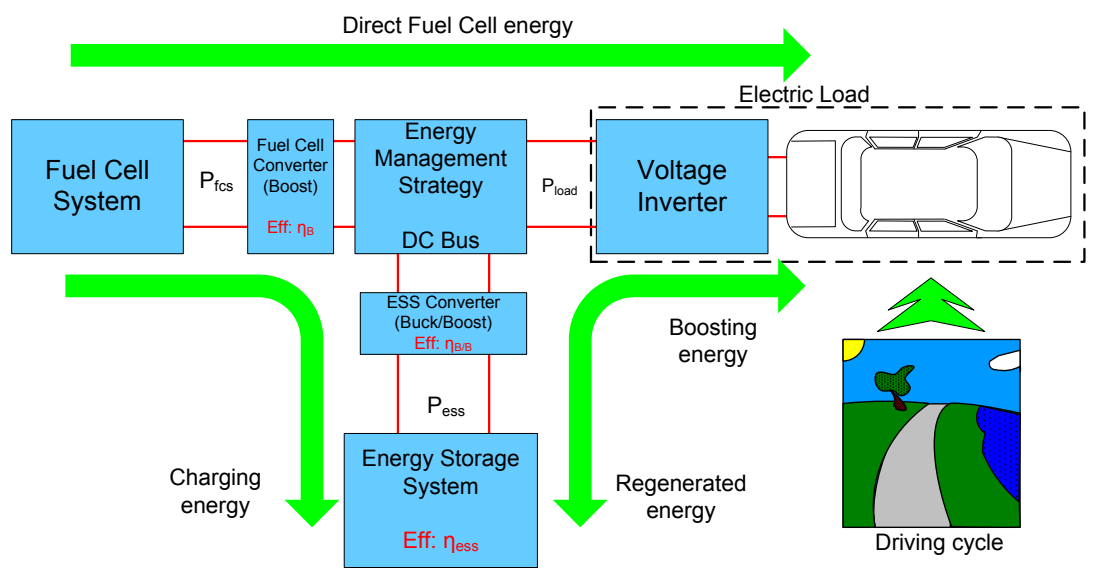

Fig. 2. Overall structure of the powertrain that drives the FCHV.

If the load power is

$$
P_{f c s, h i} \cdot \eta_{B} \leq P_{\text {load }}(k) \leq P_{f c s, \max } \cdot \eta_{B},
$$

and the $S o E$ is

$$
S_{o} E_{l o} \leq \operatorname{SoE}(k) \leq S o E_{h i},
$$

then, the FCS is operated in load following mode:

$$
P_{f c s}(k)=P_{\text {load }}(k) / \eta_{B}
$$

and $P_{\text {ess }}(k)$ is as indicated in (10) or (9).

On the other hand, if

$$
P_{\text {load }}(k) \geq P_{f c s, \max } \cdot \eta_{B} \quad \text { and } \quad S o E(k) \leq S o E_{h i}
$$

or

$$
\operatorname{SoE}(k) \leq S_{o} E_{l o},
$$

then, the $F C S$ is operated at its maximum power:

$$
P_{f c s}(k)=P_{f c s, \max },
$$

and $P_{e s s}(k)$ is as indicated in (9). If, on the contrary

$$
P_{l o a d}(k) \leq P_{f c s, l o} \cdot \eta_{B} \quad \text { and } \quad S o E(k) \geq S o E_{l o},
$$

or

$$
S o E(k) \geq S o E_{h i}
$$

then, the $F C S$ is operated at its lower operating point:

$$
P_{f c s}(k)=P_{f c s, l o},
$$

and $P_{\text {ess }}(k)$ is as in (10). Additionally, if $P_{\text {load }}(k)=$ $0 \forall t \in\left[k_{1}, k_{2}\right]$ with $\left(k_{2}-k_{1}\right)>T_{o f f}$, and, $\operatorname{SoE}(k)>S o E_{h i}$ with $k>k_{2}$, then, the FCS is turned off to avoid unnecessary hydrogen consumption because the parasitic losses in the FCS. The operation of the EMS is shown in Fig. 3 where can be distinguished four operating zones.

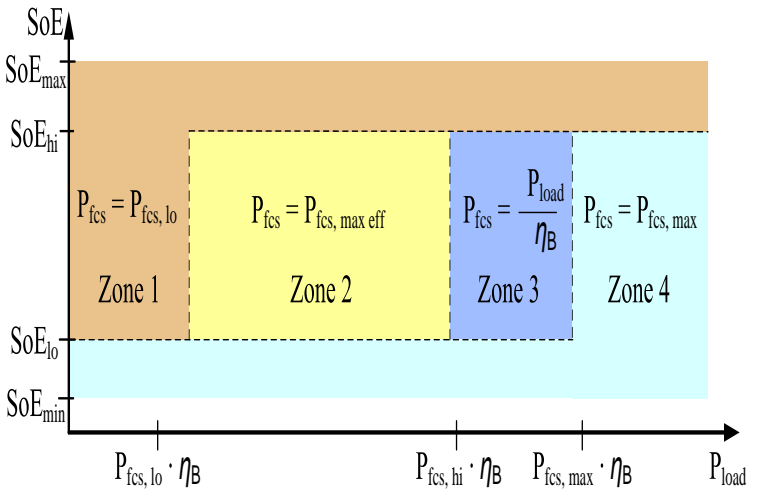

Fig. 3. Operation of the EMS.

\section{Application Results}

The proposed control structure have been tested with four standard driving cycles: the New European Driving Cycle (NEDC), the Urban Dynamometer Driving Schedule (UDDS) (also known as Federal Urban Driving Schedule (FUDS)), the Federal Test Procedure (FTP), and the Highway Fuel Economy Cycle (HWFET). To illustrate the results, Fig. 4 to 9 show the results using NEDC cycle.

The calculation of the power that the vehicle demands is done exploiting a detailed model of the FCHV developed using ADVISOR. The toolbox ADVISOR (ADvanced VehIcle SimulatOR) has been developed by the National Renewable Energy Laboratory with the aim of analyzing the performance and fuel economy of conventional, electric, and hybrid vehicles $[14,15]$. The FCHV in study corresponds to the parameters of a small vehicle described in Table I with vehicle total mass of $1380 \mathrm{~kg}$.

Fig. 5 shows the evolution of the power bus, which 
TABLE I.

VEHICLE SPECIFICATION IN THE CASE OF STUDY

\begin{tabular}{|l|c|c|}
\hline Specification & Value & Unit \\
\hline Vehicle total mass & 1380 & $\mathrm{~kg}$ \\
Vehicle mass & 882 & $\mathrm{~kg}$ \\
Frontal area & 2 & $\mathrm{~m}^{2}$ \\
Drag coefficient & 0.335 & - \\
Coefficient of rolling friction & 0.009 & - \\
Air density & 1.2 & $\mathrm{~kg} \cdot \mathrm{m} 3$ \\
\hline
\end{tabular}

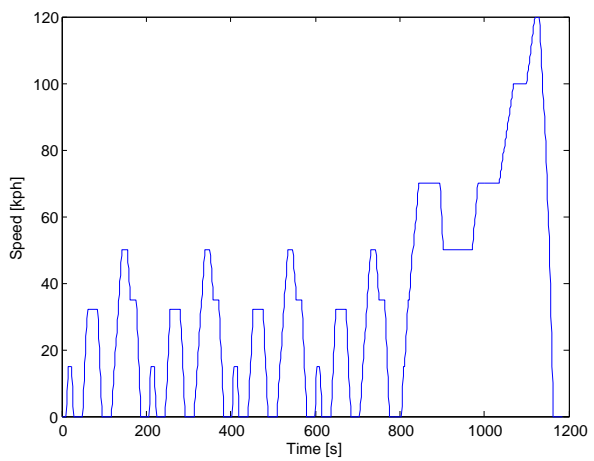

Fig. 4. Speed profile and power split

is the required power to the DC bus to fulfil the NEDC cycle shown in Fig. 4. In Fig. 5, it is also shown the evolution of the net fuel cell power and the supercapacitor power in accordance to the energy management strategy described in Section III-B. The supercapacitor SoE variation, as a result of the bidirectional supercapacitor power flow, is shown in Fig. 6. It is remarkable that it is possible to meet the load power in the four driving cycles with a $15-k W$ FCS that is significantly lower to the corresponding in the pure fuel cell case with no hybridization $(37.5 \mathrm{~kW})$, an advantage that is translated in a reduction in the production costs. This is possible thanks to the ESS power assistance and the proposed energy management strategy.

On the other hand, in Fig. 7, it can be seen how the EMS switches the zones according to control algorithm previously described and the load requirements. The manipulated variable, the compressor motor voltage, is also plotted in Fig. 8. Finally, in Fig. 9 can be seen than almost perfect control is achievable for the air supply flow compared to the optimal setpoint and trajectory, which shows that the control objective is satisfactorily fulfilled.

\section{CONCLUSIONS}

From the simulated results is concluded that the improved APCWRF combined with feed-forward action is able to give a good alternative control structure

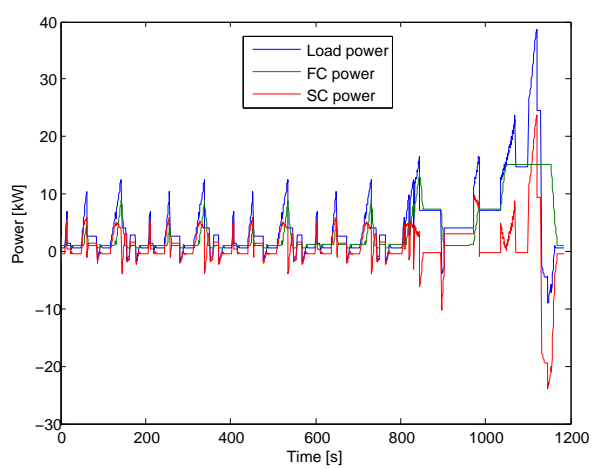

Fig. 5. Power split using the EMS

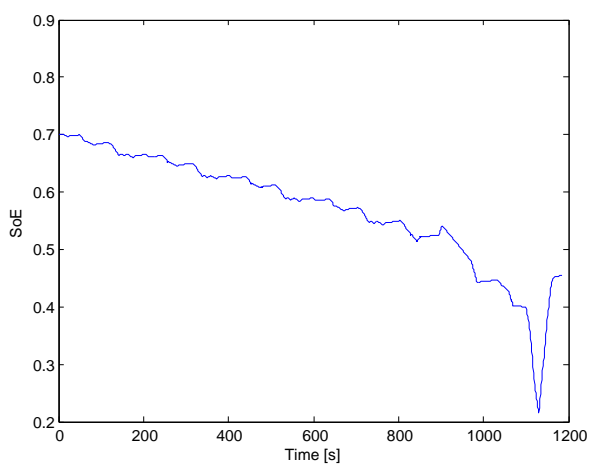

Fig. 6. State of Energy SoE of the supercapacitors

for the FCHV. In fact, the APCWRF structure allows working efficiently even though the sudden changes in the load such as in a FCHV. Moreover, with the the APCWRF it is possible to keep the controlled and performance variables close to the optimal values despite the load changes imposed by the three driving cycles analyzed here.

\section{ACKNOWLEDGEMENT}

M. Basualdo, L. Nieto, D. Zumoffen and D. Feroldi wants to acknowledge the financial support of CONICET and ANPCyT under PICT $\mathrm{N}^{\circ}$ 20060226. J. Riera wants to acknowledge the financial support of CICYT under DPI2007-62966 of the Spanish Government.

\section{REFERENCES}

[1] F. Zenith and S. Skogestad. Control of fuel cell power output. Journal of Process Control, 17:333-347, 2007.

[2] S. Caux, J. Lachaize, M. Fadel, P. Shott, and L. Nicod. Modelling and control of a fuel cell system and storage elements in transport applications. Journal of Process Control, 15:481491, 2005. 


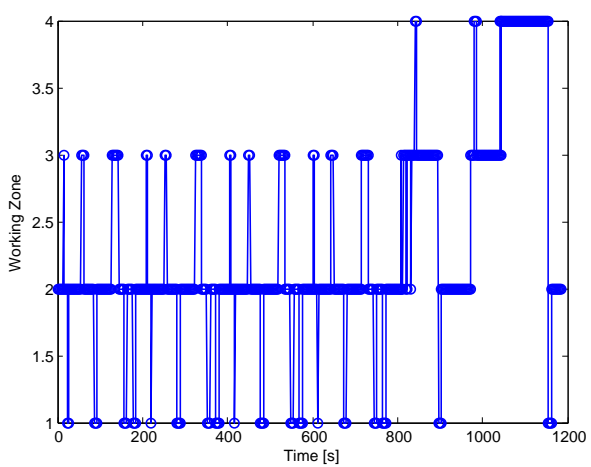

Fig. 7. Working zone of the EMS

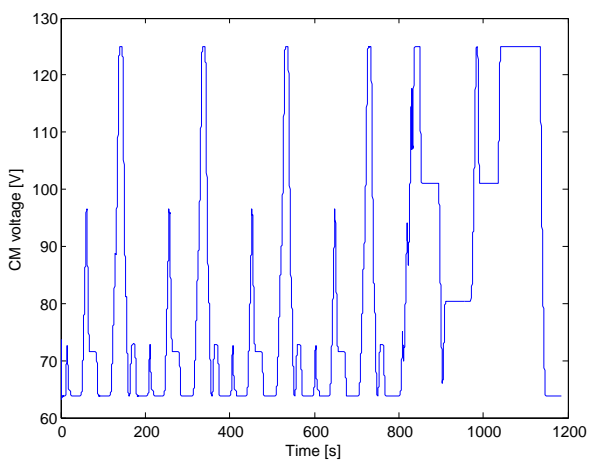

Fig. 8. Compressor motor voltage

[3] P. Thounthong, S. Raël, and B. Davat. Control strategy of fuel cell/supercapacitors hybrid power sources for electric vehicle. Journal of Power Sources, 158:806-814, 2006.

[4] K. S. Jeong, W. Y. Lee, and C. S. Kim. Energy management strategies of a fuel cell/battery hybrid system using fuzzy logics. Journal of Power Sources, 145:319-326, 2005.

[5] David Zumoffen and Marta Basualdo. Advanced Control for Fuel Cells Connected to a DC/DC Converter and an Electric Motor. In Computers and Chemical Engineering, pages 643-655, 2010.

[6] S. Biset, L. Nieto Degliuomini, M. Basualdo, V. M. Garcia, and M. Serra. Analysis of the control structures for an integrated ethanol processor for proton exchange membrane fuel cell systems. Journal of Power Sources, 2009.

[7] M. Grujicic, K.M. Chittajallu, E.H. Law, and J.T. Pukrushpan. Model-based Control Strategies in the Dynamic Interaction of Air supply and Fuel cell. Journal of Power and Energy, 218(7):487-499, 2004.

[8] DieselNet. Emission test cycles. Online, 2005.

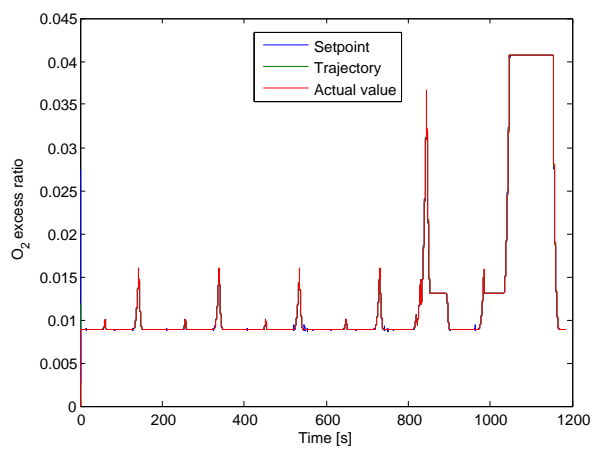

Fig. 9. Oxygen flow in the cathode

http:// www.dieselnet.com / standards / cycles/.

[9] JT Pukrushpan and A.G.H. Peng. Modeling and control for PEM fuel cell stack system. American Control Conference. Proceedings of the 2002, 4, 2002.

[10] MA Jordan, MS Basualdo, and D. Zumoffen. An approach to improve the performance of adaptive predictive control systems: theory, simulations and experiments. International Journal of Control, 79(10):1216-1236, 2006.

[11] D. Zumoffen, M. Basualdo, M. Jordan, and A. Ceccatto. Robust adaptive predictive faulttolerant control linked with fault diagnosis system applied on a nonlinear chemical process. In 2006 45th IEEE Conference on Decision and Control, pages 3512-3517, 2006.

[12] D. Zumoffen, M. Basualdo, M. Jordán, and A. Ceccatto. Robust adaptive predictive faulttolerant control integrated to a fault-detection system applied to a nonlinear chemical process. Ind. Eng. Chem. Res, 46(22):7152-7163, 2007.

[13] D. Feroldi, M. Serra, and J. Riera. Energy management strategies based on efficiency map for fuel cell hybrid vehicles. Journal of Power Sources, 2009.

[14] K. Wipke, M. Cuddy, and S. Burch. Advisor 2.1: A user-friendly advanced powertrain simulation using a combined backward/forward approach. IEEE Transactions on Vehicular Technology, 48:1751-1761, 1999.

[15] T. Markel, A. Brooker, T. Hendricks, V. Johnson, K. Kelly, B. Kramer, M. O'Keefe, S. Sprik, and K. Wipke. Advisor: a system analysis tool for advanced vehicle modeling. J. of Power Sources, 110:255-266, 2002. 